\title{
Interference and Coverage Analysis in Coexisting RF and Dense TeraHertz Wireless Networks
}

\author{
Javad Sayehvand and Hina Tabassum, Senior Member IEEE
}

\begin{abstract}
This paper develops a stochastic geometry framework to characterize the statistics of the downlink interference and coverage probability of a typical user in a coexisting terahertz (THz) and radio frequency (RF) network. We first characterize the exact Laplace Transform (LT) of the aggregate interference and coverage probability of a user in a THz-only network. Then, for a coexisting RF/THz network, we derive the coverage probability of a typical user considering biased received signal power association (BRSP). The framework can be customized to capture the performance of a typical user in various network configurations such as THz-only, opportunistic RF/THz, and hybrid RF/THz. In addition, asymptotic approximations are presented for scenarios where the intensity of $\mathrm{THz}$ BSs becomes large or molecular absorption coefficient in $\mathrm{THz}$ approaches to zero. Numerical results demonstrate the accuracy of the derived expressions and extract insights related to the significance of the BRSP association compared to the conventional reference signal received power (RSRP) association in the coexisting network.
\end{abstract}

Index Terms-Terahertz (THz), interference, coverage probability, stochastic geometry, Poisson point process (PPP).

\section{INTRODUCTION}

The potential of using higher frequency spectrum such as terahertz $(\mathrm{THz})$ in the sixth generation $(6 \mathrm{G})$ wireless networks is evident [1]. THz frequencies offer ample spectrum, multi Gigabit-per-second (Gbps) data rates, and highly secure transmissions. Nonetheless, compared to conventional radio frequency $(\mathrm{RF}), \mathrm{THz}$ transmissions incur high propagation loss mainly due to molecular absorption resulting from water vapors or oxygen molecules, thus significantly limiting the communication distance. $\mathrm{THz}$ spectrum is thus complementary to conventional RF spectrum. Recent advancements have made it possible to mount $\mathrm{THz}$ transceivers on smart phones, e.g., Fujitsu who introduced a compact $300 \mathrm{GHz}$ transceiver capable of $20 \mathrm{Gbps}$ data stream. [2].

To date, most of the research works considered analyzing the performance of a given $\mathrm{THz}$ transmission link [1] or THz-only network [3]-[5]. For instance, the authors in [1] derived a closed-form expression of the outage probability and ergodic capacity considering a $\mathrm{THz}$ wireless fiber extender system (i.e., a single transmission link) with ideal and nonideal RF front-end. Using tools from stochastic geometry and considering interference limited regime, the authors derived the mean interference in a THz-only network [3]. However, the closed-form expression of the mean interference was neither applicable for a general case, nor the expression was applied to the outage analysis. Instead, the authors approximate the distribution of the interference with log-logistic distribution to overcome the intractable outage calculation. Nevertheless, as the authors mentioned, the use of log-logistic approximation

J. Sayehvand and H. Tabassum are with the York University, Canada (e-mail: jsayehvand@cse.yorku.ca and hina@eecs.yorku.ca). This work is supported by the Discovery Grant from the Natural Sciences and Engineering Research Council of Canada. might not be accurate in all scenarios. Similarly, [4] considered Taylor expansion and calculated the approximations for mean and variance of signal-to interference-plus-noise ratio (SINR). In [5], the authors analyzed the reliability and endto-end latency considering a THz-only network with finite number of BSs. The interference was approximated with a normal distribution. The authors in [6] derived the approximate coverage probability in a single-tier network, where BSs can use either RF or $\mathrm{THz}$.

To our best knowledge, none of the aforementioned research works presented a comprehensive analytic framework to characterize the exact interference statistics and coverage probability of users in a THz-only network or a coexisting two-tier RF and dense $\mathrm{THz}$ network

Using stochastic geometry, this paper characterizes the statistics of the downlink interference and rate coverage probability of a typical user in a coexisting $\mathrm{RF} / \mathrm{THz}$ network. The proposed framework can be customized for various network configurations, including (i) $\mathrm{THz}$-only network where only TBSs exist and users associate to their nearest BS, (ii) opportunistic $\mathrm{RF} / \mathrm{THz}$ network where a user associates to the BS with maximum biased received signal power (BRSP) 1 , and (iii) Hybrid network where a user associates to both nearest RF and TBSs. We first characterize the exact Laplace Transform (LT) of the aggregate interference and coverage probability of a user in a THz-only network. Then, we derive the coverage probability of a typical user in a coexisting network. Asymptotic approximations are presented for large intensity of TBSs or small molecular absorption coefficients. Numerical results show the significance of BRSP over conventional reference signal received power (RSRP) association in a coexisting network and validate the derived expressions.

\section{SySTEM MODEL AND ASSUMPTIONS}

We consider a two-tier downlink network composed of RF SBSs and TBSs as well as users' devices. The locations of the conventional RF SBSs and TBSs are modeled as a twodimensional (2D) homogeneous Poisson point processes (PPP) $\boldsymbol{\Phi}_{R}$ and $\boldsymbol{\Phi}_{T}$ with intensities $\lambda_{R}$ and $\lambda_{T}$, respectively. The locations of the users follow independent homogeneous PPP $\Phi_{u}$ with intensity $\lambda_{u}$. Each user measures the channel quality from each BS and then associate to the chosen BS according to a predefined association mechanism. The BSs serve associated users in orthogonal time slots or channels. We consider the performance of a typical user who is located at the origin.

1) RF Channel and SINR Model: The RF channel experiences both the channel fading and path-loss. Thus, the

\footnotetext{
${ }^{1}$ BRSP-based association is considered by $3 \mathrm{GPP}$ in Release 10 , where the users' power received from small base stations (SBSs) has been artificially increased by adding a bias in order to avoid under-utilization of SBSs [7].
} 
received signal power at the typical user can be modeled as $h(\rho)=\gamma_{R} \rho^{-\alpha} \chi$, where $\gamma_{R}=\frac{c^{2}}{\left(4 \pi f_{R}\right)^{2}}, \chi$ is the exponentially distributed channel power with unit mean from the tagged SBS, $\alpha$ is the path-loss exponent, $\rho$ is the distance of the typical user to the serving SBS, $f_{R}$ is the RF carrier frequency in $\mathrm{GHz}$, and $c=3 \times 10^{8} \mathrm{~m} / \mathrm{s}$ is the speed of light. We consider SBSs equipped with omni-directional antennas. Therefore, the SINR of a typical user can be modeled as:

$$
\mathrm{SINR}_{R}=\frac{P_{R} \gamma_{R} \rho_{0}^{-\alpha} \chi_{0}}{N_{0}^{R}+I_{\mathrm{agg}}^{R}},
$$

where $\chi_{0}$ is the fading channel power of the typical user from the desired SBS, $P_{R}$ is the transmit power of the SBSs, $N_{0}^{R}$ is the thermal noise at the receiver, $I_{\text {agg }}^{R}=$ $\sum_{i \in \Phi_{R} \backslash 0} P_{R} \gamma_{R} \rho_{i}^{-\alpha} \chi_{i}$ is the aggregate interference at the typical user from the interfering SBSs, $\rho_{i}$ is the distance between the $i$-th interfering SBS and the typical user, and $\chi_{i}$ is the fading channel power from the $i$-th interfering SBS.

2) $\mathrm{THz}$ Channel and SINR Model: Due to high molecular absorption and the dense deployment, the LoS transmissions are dominant than the NLoS transmissions. Therefore, following [3], [5], [8], [9], we model the line-of-sight (LoS) channel powet between users and TBSs as $h(r)=$ $\frac{c^{2}}{\left(4 \pi f_{T}\right)^{2}} \frac{\exp \left(-k_{a}(f) r\right)}{r^{2}}$, where $k_{a}(f)$ is the molecular absorption coefficient, $r$ is the distance between the transmitter and receiver, $f_{T}$ is the operating $\mathrm{THz}$ frequency, $c$ is the speed of light, and $G_{\mathrm{tx}}^{\mathrm{T}}(\theta)$ as well as $G_{\mathrm{rx}}^{\mathrm{T}}(\theta)$ are the directional transmitter and receiver antenna gains, respectively. The directional antennas are modeled as [10]:

$$
G_{q}^{T}(\theta)= \begin{cases}G_{q}^{(\max )} & |\theta| \leq w_{q} \\ G_{q}^{(\min )} & |\theta|>w_{q}\end{cases}
$$

where $q \in\{\mathrm{tx}, \mathrm{rx}\}, \theta \in[-\pi, \pi)$ is the angle of the boresight direction, $w_{q}$ is the main lobe beamwidth, $G_{q}^{(\max )}$ and $G_{q}^{(\min )}$ are beamforming gains of the main and side lobes, respectively. The typical user and its desired TBS align such that their main lobes coincide through beam alignment techniques. The alignment between the typical user and an interferer is defined as a random variable $D$, which can take values in $\left\{G_{\mathrm{tx}}^{(\max )} G_{\mathrm{rx}}^{(\max )}, G_{\mathrm{tx}}^{(\max )} G_{\mathrm{rx}}^{(\min )}, G_{\mathrm{tx}}^{(\min )} G_{\mathrm{rx}}^{(\max )}, G_{\mathrm{tx}}^{(\min )} G_{\mathrm{rx}}^{(\min )}\right\}$, and the corresponding probability for each case is $F_{\mathrm{tx}} F_{\mathrm{rx}}$, $F_{\mathrm{tx}}\left(1-F_{\mathrm{rx}}\right),\left(1-F_{\mathrm{tx}}\right) F_{\mathrm{rx}}$, and $\left(1-F_{\mathrm{tx}}\right)\left(1-F_{\mathrm{rx}}\right)$, where $F_{\mathrm{tx}}=\frac{\theta_{\mathrm{tx}}}{2 \pi}$ and $F_{\mathrm{rx}}=\frac{\theta_{\mathrm{rx}}}{2 \pi}$, respectively. Assuming that the main lobe typical user's receiver is coinciding with that of its desired TBS, its SINR can be formulated as follows:

$$
\mathrm{SINR}_{T}=\frac{P_{\mathrm{T}} G_{\mathrm{tx}}^{(\max )}(\theta) G_{\mathrm{rx}}^{(\max )}(\theta) \frac{c^{2}}{\left(4 \pi f_{T}\right)^{2}} \frac{\exp \left(-k_{a}(f) r\right)}{r^{2}}}{N_{0}^{T}+I_{\mathrm{agg}}^{T}}
$$

where $I_{\text {agg }}^{T}=\sum_{\mathrm{i} \in \Phi_{T} \backslash 0} P_{\mathrm{T}} D_{i} h\left(r_{i}\right)$ is the aggregate interference at the typical user by their maximum gain, $r_{i}$ is the distance of the typical user to the interfering TBSs. For brevity, we define $\gamma_{T}=G_{\mathrm{tx}}^{(\max )}(\theta) G_{\mathrm{rx}}^{(\max )}(\theta) \frac{c^{2}}{\left(4 \pi f_{T}\right)^{2}}$. We assume that the interferers' main lobe coincides with the users' main lobe 3 with the probability of $F=F_{\mathrm{tx}} F_{\mathrm{rx}}$, and thus, $D=G_{\mathrm{tx}}^{(\max )}(\theta) G_{\mathrm{rx}}^{(\max )}(\theta)$. Also, $P_{T}$ is the transmit power of the TBSs, and $N_{0}^{T}$ denotes the thermal noise and the noise resulted from the molecular absorption which is considered as negligible in dense $\mathrm{THz}$ networks.

\section{Coverage Probability in THz-Only Network}

In this section, we derive the LT of the aggregate interference and the coverage probability experienced by a typical user in a THz-only network with nearest BS association.

Lemma 1. Conditioned on the distance of a typical user from the serving TBS, the LT of the aggregate interference, at a typical device in $\mathrm{THz}$ network, can be derived as follows:

$\mathcal{L}_{I_{\text {agg }}^{T} \mid r}(s)=\exp \left(2 \pi \lambda_{T} \sum_{l=1}^{\infty} \frac{\left.\left(-s \gamma_{T} F P_{\mathrm{T}}\right)^{l} \Gamma\left(2-2 l, l k_{a}(f) r\right)\right)}{\left(l k_{a}(f)\right)^{2-2 l} l !}\right)$.

\section{Proof. See Appendix A.}

We define the rate coverage probability as the probability with which a typical user achieves the desired target rate $R_{\mathrm{th}}$. Subsequently, using $R_{\mathrm{th}}=W_{T} \log _{2}\left(1+\mathrm{SINR}\right.$ ) (where $W_{T}$ is the $\mathrm{THz}$ transmission bandwidth), the rate coverage probability can thus be given as follows:

$$
\mathcal{P}_{T}=\operatorname{Pr}\left(\operatorname{SINR}_{T}>2^{\frac{R_{\mathrm{th}}}{W_{T}}}-1\right)=\operatorname{Pr}\left(\operatorname{SINR}_{T}>\tau_{T}\right) .
$$

Taking the desired signal power at the typical user $S(r)=$ $P_{T} \gamma_{T} \frac{\exp \left(-k_{a}(f) r\right)}{r^{2}}$ and using the Gil-Pelaez inversion theorem [11], $\mathcal{P}_{T}$ can be derived as follows:

$$
\begin{aligned}
& \mathcal{P}_{T}=\operatorname{Pr}\left(\frac{S(r)}{N_{0}^{T}+I_{\mathrm{agg}}^{T}}>\tau_{T}\right)=\operatorname{Pr}\left(S(r)>\tau_{T} I_{\mathrm{agg}}^{T}+\tau_{T} N_{0}^{T}\right), \\
& =\mathbb{E}_{r}\left[\frac{1}{2}-\frac{1}{\pi} \int_{0}^{\infty} \frac{\operatorname{Im}\left[\phi_{\Omega \mid r}(\omega) e^{j \omega \tau_{T} N_{0}^{T}}\right]}{\omega} d \omega\right] \\
& =\frac{1}{2}-\frac{1}{\pi} \int_{0}^{\infty} \frac{\operatorname{Im}\left[\phi_{\Omega}(\omega) e^{j \omega \tau_{T} N_{0}^{T}}\right]}{\omega} d \omega
\end{aligned}
$$

where $\operatorname{Im}(\cdot)$ is the imaginary part of $\phi_{\Omega}(\cdot), \Omega=S(r)-$ $\tau_{T} I_{\text {agg }}^{T}$, and $\phi_{\Omega}(w)=\mathbb{E}\left[e^{-j \omega \Omega}\right]$ is the characteristic function $(\mathrm{CF})$ of $\Omega$ given as follows:

$$
\phi_{\Omega}(\omega)=\mathbb{E}_{r}\left[\phi_{\Omega \mid r}(\omega)\right]=\mathbb{E}_{r}\left[e^{-j \omega S(r)} \mathcal{L}_{I_{\text {agg }}^{T} \mid r}\left(-j \omega \tau_{T}\right)\right],
$$

where $\mathcal{L}_{I_{\text {agg }}^{T} \mid r}$ is given in Lemma 1. Gil-Pelaez inversion is applicable to the $\mathrm{CF}$ of any random variable and has been proved useful in a wide variety of wireless applications [12].

\section{Coverage in Coexisting RF/THz Network}

In the coexisting network, the user can either associate to a given SBS or TBS based on the maximum BRSP with a probability termed as association probability.

${ }^{3}$ For simplicity, we consider negligible side lobe gains. However, the framework can be extended by averaging over variable $D$ and considering all four possible interference components in $I_{\mathrm{agg}}^{\mathrm{T}}$ with different antenna gains. These four interference variables are independent and their LTs can be given using Lemma 1. $\mathcal{L}_{I_{\text {agg }}^{T} \mid r}(s)$ can thus be given as the product of their LTs.

\footnotetext{
${ }^{2}$ The consideration of NLoS with accurate reflection, scattering, and diffraction models deserves a separate study and has been left for future investigation.
} 
Given the received powers from TBSs and SBSs as $P_{r}^{\mathrm{THz}}=$ $P_{T} \gamma_{T} \times \frac{\exp \left(-k_{a}(f) r\right)}{r^{2}}$ and $P_{r}^{\mathrm{RF}}=P_{R} \gamma_{R} \times \rho^{-\alpha}$, respectively, the probability of association to TBS can be derived as follows:

$$
\begin{aligned}
& \mathcal{P}_{A_{T}}=\mathbb{E}_{r}\left[\operatorname{Pr}\left[B_{T} P_{r}^{\mathrm{THz}}>P_{r}^{\mathrm{RF}}\right]\right], \\
& =\mathbb{E}_{r}\left[\operatorname{Pr}\left[P_{T} B_{T} \gamma_{T} \frac{\exp \left(-k_{a}(f) r\right)}{r^{2}}>P_{R} \gamma_{R} \rho^{-\alpha}\right]\right], \\
& \stackrel{(a)}{=} \mathbb{E}_{r}\left[\exp \left(-\pi \lambda_{R}\left(K r^{2} \exp \left(k_{a}(f) r\right)\right)^{\frac{2}{\alpha}}\right)\right],
\end{aligned}
$$

where $K=\frac{P_{R} \gamma_{R}}{B_{T} P_{T} \gamma_{T}}, B_{T}>1$ is the bias value to encourage association with THz layer, $0 \leq B_{T}<1$ encourages association to RF layer, $B_{T}=1$ yields conventional RSRP and (a) follows from the null property of PPP $\Phi_{R}$. This property implies that given a tier of RF SBSs with intensity $\lambda_{R}$, the probability that no RF BSs are closer to typical user than the distance $z$ is $\mathbb{P}[\rho \geq z]=\exp \left(-\pi \lambda_{R} z^{2}\right)$.

Taking the expectation, the association probability of a typical user to TBSs can be derived as in the following lemma.

Lemma 2. Given that the user associates with the layer that provides the maximum BRSP, the probability of association to the $\mathrm{THz}$ layer is given as follows:

$$
\mathcal{P}_{A_{T}}=\sum_{j=0}^{\infty} \frac{(-1)^{j} \Gamma\left[v_{j}\right] \delta_{T, j}}{\left(2 \beta_{T}\right)^{\frac{v_{j}}{2}} j !} \exp \left(\frac{-\eta_{j}}{8 \beta_{T}}\right) D_{-v_{j}}\left(\frac{-\eta_{j}}{\sqrt{2 \beta_{T}}}\right),
$$

where $\beta_{T}=\pi \lambda_{T}, \delta_{T, j}=2 \pi \lambda_{T}\left(\pi \lambda_{R} K^{\frac{2}{\alpha}}\right)^{j}, \nu_{j}=\frac{4 j}{\alpha}+2$, $\eta_{j}=-\frac{2 j k(f)}{\alpha}$, and $D_{\nu}(z)$ is the parabolic cylinder function $(P C F)$ ( $[13], E q .9 .240)$. Clearly, the probability that a device associates to the RF layer is given by

$$
\mathcal{P}_{\mathrm{A}_{\mathrm{R}}}=1-\mathcal{P}_{\mathrm{A}_{\mathrm{T}}} \text {. }
$$

\section{Proof. See Appendix B.}

As the future networks will be highly dense, the density of TBSs can be very high $\left(\lambda_{T} \rightarrow \infty\right)$. Also, for indoor applications [5], the absorption loss can approach zero $\left(k_{a}(f) \rightarrow 0\right)$. By demonstrating the limit of $\infty$, we mean that the intensity can be quite large but may not be close to infinity. In both special cases, the association probability can be simplified as.

Corollary 1. When $\lambda_{T} \rightarrow \infty \Longrightarrow z \rightarrow 0$ and $k_{a} \rightarrow 0 \Longrightarrow$ $z \rightarrow 0$, the argument of $D_{\nu}(z)$ in Lemma 2 tends to zero. For $z=0, D_{\nu}(z)$ will be simplified to $\frac{\sqrt{\pi}}{2^{\frac{1}{2} b+\frac{1}{4}} \Gamma\left(\frac{3}{4}+\frac{1}{2} b\right)}$, where $b=-\frac{1}{2}-\nu$ and $\Gamma(z)$ is the gamma function ( [13], Eq. 8.31). As a result, $\mathcal{P}_{\mathrm{A}_{\mathrm{T}}}$ in lemma 2 can be simplified as:

$$
\mathcal{P}_{\mathrm{A}_{\mathrm{T}}}=\sum_{j=0}^{\infty} \frac{\sqrt{\pi}(-1)^{j} \delta_{j}(2 \beta)^{-\frac{v_{j}}{2}} \Gamma\left[v_{j}\right]}{2^{\frac{1}{2} b_{j}+\frac{1}{4}} \Gamma\left(\frac{3}{4}+\frac{1}{2} b_{j}\right) j !} \exp \left(\frac{-\eta_{j}}{8 \beta}\right),
$$

where $b_{j}=-\frac{1}{2}-\nu_{j}$.

Since a typical user can associate with either $\mathrm{RF}$ or $\mathrm{THz}$ layer, the total coverage probability can be calculated as:

$$
\mathbb{C}=\mathcal{P}_{\mathrm{A}_{\mathrm{T}}} \mathbb{P}_{C_{\mathrm{T}}}+\mathcal{P}_{\mathrm{A}_{\mathrm{R}}} \mathbb{P}_{\mathcal{C}_{\mathrm{R}}}
$$

where $\mathcal{P}_{\mathrm{A}_{\mathrm{T}}}$ and $\mathcal{P}_{\mathrm{A}_{\mathrm{R}}}$ are defined in Lemma 2. Also, $\mathbb{P}_{\mathrm{C}_{\mathrm{T}}}$ and $\mathbb{P}_{C_{\mathrm{R}}}$ refer to the coverage probability conditioned that the typical user associates to a given TBS and RF SBS, respectively. Since the TBSs and RF SBSs are distributed according to different PPPs, the distance of a typical user to its serving BS depends on the tier to which the user is associated. Subsequently, the distribution of the distance of the typical user to its serving BS in $k$-th tier can be given as:

Lemma 3. The distribution of the distance of a typical user if it is tagged to the $\mathrm{THz}$ layer and SBS layer can be given, respectively, as follows:

$$
\begin{gathered}
f_{\hat{X}_{T}}(\hat{x})=\frac{2 \pi \lambda_{T} \hat{x}}{\mathcal{P}_{\mathrm{A}_{\mathrm{T}}}} \exp \left(-\pi \lambda_{R}\left(K \hat{x}^{2}\right)^{2 / \alpha} e^{2 k_{a}(f) \hat{x} / \alpha}-\pi \lambda_{T} \hat{x}^{2}\right), \\
f_{\hat{X}_{R}}(\hat{x}) \approx \frac{2 \pi \lambda_{R} \hat{x}}{\mathcal{P}_{\mathrm{A}_{\mathrm{R}}}} \exp \left(-\pi \lambda_{T}\left(\frac{K \hat{x}^{\alpha}}{\pi}\right)^{\frac{1}{2+\mu}}-\pi \lambda_{R} \hat{x}^{2}\right),
\end{gathered}
$$

where $\mu$ is a factor defined in the proof.

\section{Proof. See Appendix C.}

Lemma 4. The calculation of $\mathbb{P}_{\mathrm{C}_{\mathrm{T}}}$ can be performed using the Gil-Pelaez inversion theorem as given in (5) where

$\phi_{\Omega}(\omega)=\mathbb{E}_{\hat{X}_{T}}\left[\phi_{\Omega \mid \hat{X}_{T}}(\omega)\right]=\mathbb{E}_{\hat{X}_{T}}\left[e^{-j \omega S(\hat{x})} \mathcal{L}_{I_{\mathrm{agg}}^{T} \mid \hat{X}_{T}}\left(-j \omega \tau_{T}\right)\right]$, and the PDF of $\hat{X}_{T}$ is given in Lemma 3 Considering the interference limited regime, the conditional coverage probability of the user when associated to RF layer can be given as:

$$
\begin{aligned}
& \mathbb{P}_{C_{\mathrm{R}}}=\operatorname{Pr}\left[\chi_{0}>\tau_{R} P_{R}^{-1} \gamma_{R}^{-1} \hat{x}^{\alpha} I_{a g g}^{R}\right], \\
& =\mathbb{E}_{I_{a g g}^{R}, \hat{x}}\left[\exp \left(-\tau_{R} P_{R}^{-1} \gamma_{R}^{-1} \hat{x}^{\alpha} I_{a g g}^{R}\right)\right], \\
& =\int_{0}^{\infty} \mathcal{L}_{I_{a g g}^{R}}\left(\tau_{R} P_{R}^{-1} \gamma_{R}^{-1} \hat{x}^{\alpha}\right) f_{\hat{X}_{R}}(\hat{x}) d \hat{x},
\end{aligned}
$$

where $\mathcal{L}_{I_{\text {agg }}^{R}}\left(\tau_{R} P_{R}^{-1} \gamma_{R}^{-1} \hat{x}^{\alpha}\right)=\exp \left(-\pi \hat{x}^{2} \lambda_{R} \mathcal{Z}\left(\tau_{R}, \alpha\right)\right)$, $\mathcal{Z}\left(\tau_{R}, \alpha\right) \stackrel{2 \tau_{R}}{\alpha-2} F_{1}\left[1,1-\frac{2}{\alpha} ; 2-\frac{2}{\alpha} ;-\tau_{R}\right]$, and ${ }_{2} F_{1}[\cdot]$ is Gauss Hypergeometric function [13].

Remark 1. The coverage probability of hybrid $\mathrm{RF} / \mathrm{THz}$ scheme can be given as $\mathbb{C}_{\mathrm{Hybrid}}=$ $1-\left(1-\mathcal{P}_{T}\right)\left(1-\mathcal{P}_{R}\right)$, where $\mathcal{P}_{T}$ is given in Eq. (4), $\mathcal{P}_{R}=2 \pi \lambda_{R} \int_{0}^{\infty} \rho \mathcal{L}_{I_{a g g}^{R}}\left(\tau_{R} P_{R}^{-1} \gamma_{R}^{-1} \rho^{\alpha}\right) \exp \left(-\pi \lambda_{R} \rho^{2}\right) d \rho$ is the coverage probability of the $R F$-only network and $\mathcal{L}_{I_{\text {agg }}^{R}}(\cdot)$ is given in Lemma 4.

\section{NumERICAL RESUltS AND Discussions}

Unless stated otherwise, the simulation parameters are listed herein. Users are distributed within a circular disc of radius $100 \mathrm{~m}$. The antenna gains of TBSs $G_{\mathrm{tx}}^{T}$ and $G_{\mathrm{rx}}^{T}$ are set as 25 $\mathrm{dB}$. The transmit powers of TBSs and RF SBSs are $1 \mathrm{~W}$. Three values for $k_{a}(f)$ are considered, i.e., $0.05,0.1$, and $0.2 \mathrm{~m}^{-1}$ with $1 \%$ of water vapor molecules. These absorption values are chosen from the realistic database and their corresponding central frequencies are $1.0 \mathrm{THz}, 1.5 \mathrm{THz}$, and $1.8 \mathrm{THz}$, respectively [14] [15]. The desired rate threshold is taken as 5 Gbps. The RF transmission frequency is set as $2.1 \mathrm{GHz}$ and $\alpha=2.5$. The RF and $\mathrm{THz}$ transmission bandwidths are set as $40 \mathrm{MHz}$ and $0.5 \mathrm{GHz}$, respectively. The intensity of RF SBS $\lambda_{R}$ is set as $0.0001 \mathrm{BSs} / \mathrm{m}^{2}$.

Fig. 1 a) depicts the LT of the aggregate interference at the typical user (averaged over large number of realizations of the desired link distance $r$ ). The theoretic results are calculated 


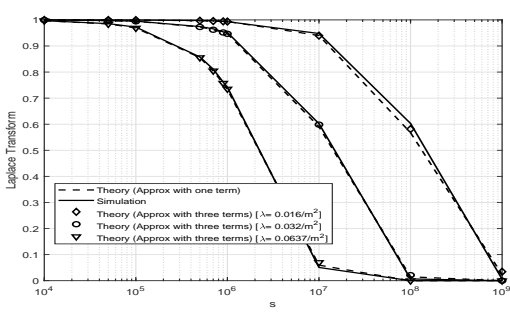

(a)

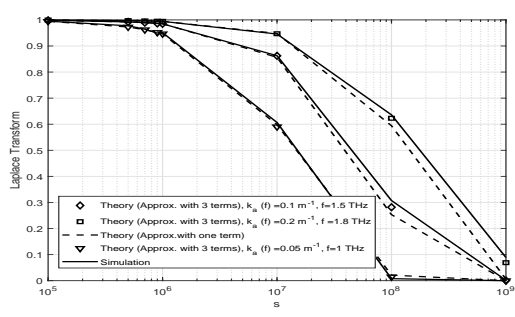

(b)

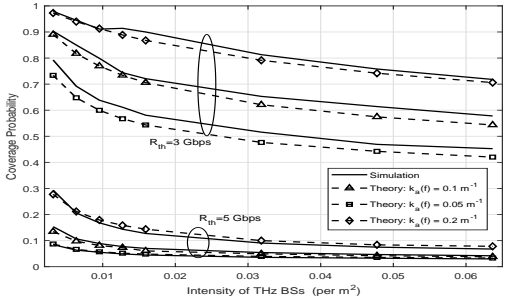

(c)

Figure 1: (a) LT of the aggregate interference as a function of the intensity of TBSs, $k_{a}(f)=0.05, f=1.0 \mathrm{THz}$. (b) LT of the aggregate interference as a function of the molecular absorption coefficients, $\lambda_{T}=0.032$ per $\mathrm{m}^{2}$. (c) Coverage probability of a user in THz-only network.

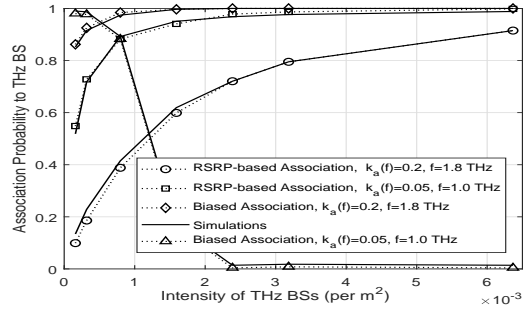

(a)

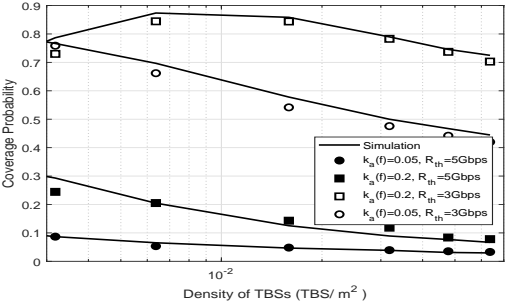

(b)

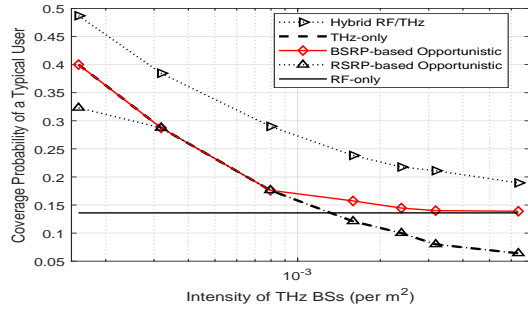

(c)

Figure 2: (a) Association probability as a function of the intensity of TBSs, $k_{a}(f)=0.2 m^{-1}, f=1.8 \mathrm{THz}, G_{T}^{T}$ and $G_{R}^{T}=15 \mathrm{~dB}, \alpha=3.6, B=[1000,100$, 1, 0.001, 0.0001, 0.0001, 0.00001] when $k_{a}(f)=0.05 \mathrm{~m}^{-1}, B=\left[10^{6}, 10^{5}, 10^{4}, 10^{4}, 10^{3}, 10^{3}, 10^{3}\right]$ when $k_{a}(f)=0.2 \mathrm{~m}^{-1}$. (b) Coverage probability in coexisting network, $\lambda_{T}=0.1 \mathrm{~m}^{-2}$. (c) Coverage probability in coexisting network, $k_{a}(f)=0.05 \mathrm{~m}^{-1}, B=\left[10^{3}, 10^{2}, 1,10^{-3}, 10^{-4}, 10^{-4}, 10^{-5}\right]$.

by taking the first three terms as well as only one term of the summation in Lemma 1. There is a close match between the theory and simulations. For a given $s$, increasing LT values mean that the aggregate interference is reducing and vice versa. In Fig. 1(a), for a given $s$, we note that increasing the intensity of TBSs, LT decreases rapidly (which implies interference increases). Similarly, for a given $s$, we note that increasing the $k_{a}(f)$ in Fig. 1(b), LT increases (which implies interference decreases due to lower absorption loss at the interfering links). The coverage probability of a user in a THz-only network is demonstrated in Fig. 11 c). The theoretical results (with first three terms of the infinite summation) show a close match with the simulations. We note that by increasing the molecular absorption coefficient, the coverage probability increases. This is in agreement with in Fig. 1(b).

The association probability to the $\mathrm{THz}$ layer is depicted in Fig. 22 a). For the RSRP-based association, the probability of user association to the $\mathrm{THz}$ layer increases with $\lambda_{T}$. However, in BRSP, the bias factor $B_{T}$ is obtained numerically to maximize the coverage probability. We note that optimal bias to TBSs decreases with the increase in $\lambda_{T}$ (which implies increased $\mathrm{THz}$ interference). Also, bias reduction is steep for low $k_{a}(f)$ (implying higher interference), whereas the reduction in bias is gradual for high values of $k_{a}(f)$ (implying lower interference). Fig. 2(b) shows the coverage probability of opportunistic RF/THz system with unit bias value. From the Fig. 2(a), it is clear that the typical user is likely to associate to the THz layer when bias is unity. Therefore, the total coverage probability is dominated by the behaviour of $\mathrm{THz}$ layer and shows the similar behaviour as THz-only network.

Fig. 2(c) compares the performance of the typical user in coexisting RF/THz network with THz-only, RF-only, and hybrid RF/THz networks. Hybrid RF/THz network outperforms all networks, since the typical user simultaneously uses both
$\mathrm{THz}$ and $\mathrm{RF}$ transmissions. That is, the additional coverage is at the expense of increased network resources. Coexisting $\mathrm{RF} / \mathrm{THz}$ with BRSP-based association maximizes the coverage by dynamically adapting to the best tier (since the bias factors are chosen to maximize the coverage) and outperforms RFonly and THz-only schemes.

\section{CONCLUSION}

We presented a unified stochastic geometry framework to characterize the performance of a user in a coexisting $\mathrm{RF} / \mathrm{THz}$ network. This work can be extended to incorporate fading by rederiving the LT of the aggregate interference with fading channel statistics. For blockages, we can follow the approach in [16]. A Boolean blockage model can be considered where the number of blockages in a link are Poisson distributed. Then, LOS probability $e^{[-(\xi r+p)]}$ (where $\xi$ and $p$ are constants) can be multiplied with $\Phi_{\Omega} \mid r(\omega)$.

\section{APPENDiX A: ProOf OF LEMMA 1}

Recall that $I_{\text {agg }}^{T}=\sum_{\mathrm{i} \in \Phi_{T} \backslash 0} P_{\mathrm{T}} D_{i} h\left(r_{i}\right)$, after averaging over $D_{i}$ the LT of the aggregate interference can be given as:

$$
\begin{aligned}
& \mathcal{L}_{I_{\mathrm{agg}}^{\mathrm{T}}}(s)=\mathbb{E}_{\Phi_{T}}\left[e^{-s I_{\mathrm{agg}}}\right]=\mathbb{E}_{\Phi_{T}}\left[e^{-s F \sum_{i \in \Phi_{T} \backslash 0} P_{\mathrm{T}} \gamma_{T} \frac{e^{-k_{a}(f) r_{i}}}{r_{i}^{2}}}\right], \\
& =\mathbb{E}_{\Phi_{T}}\left[\prod_{i \in \Phi_{T} \backslash 0} \exp \left(-s F P_{\mathrm{T}} \gamma_{T} \frac{e^{-k_{a}(f) r_{i}}}{r_{i}^{2}}\right)\right], \\
& \stackrel{(a)}{=} \exp \left(-2 \pi \lambda_{T} \int_{r}^{\infty} r_{i}\left(1-\exp \left(-s \gamma_{T} F P_{\mathrm{T}} \frac{e^{-k_{a}(f) r_{i}}}{r_{i}^{2}}\right)\right) d r_{i}\right), \\
& \stackrel{(b)}{=} \exp \left(-2 \pi \lambda_{T} \int_{r}^{\infty} \sum_{l=1}^{\infty} \frac{\left(-s \gamma_{T} F P_{\mathrm{T}}\right)^{l} \exp \left(-l k_{a}(f) r_{i}\right)}{r_{i}^{2 l-1} l !} d r_{i}\right), \\
& \left.\stackrel{(c)}{=} \exp \left(2 \pi \lambda_{T} \sum_{l=1}^{\infty} \frac{\left(-s \gamma_{T} F P_{\mathrm{T}}\right)^{l}}{\left(l k_{\alpha}(f)\right)^{2-2 l} l !} \Gamma\left(2-2 l, l k_{a}(f) r\right)\right)\right),
\end{aligned}
$$


where (a) is derived by using the probability generating functional (PGFL) with respect to $f(x)=\exp \left(-s P_{T} h\left(r_{i}\right)\right)$, (b) is derived using $\exp (-x)=\sum_{i=0}^{\infty}(-1)^{i} \frac{x^{i}}{i !}$ ( [13], Eq. 1.211), and (c) follows from the integral identity $\int \frac{\exp \left(-\beta x^{n}\right)}{x^{m}} d x=$ $-\frac{\beta^{z} \Gamma\left(-z, \beta x^{n}\right)}{n}$, and $z$ equals to $\frac{m-1}{n}$ ( [13], Eq. 2.345). Since the typical user has a distance $r$ from its serving TBS due to the nearest BS association, all interferers exist beyond $r$. Thus, the lower limit in the integral is $r$.

\section{Appendix B: Proof of Lemma 2}

The distribution of the distances between the typical user and its nearest $\mathrm{THz}$ and $\mathrm{RF}$ BSs are $f_{r}(r)=$ $2 \pi \lambda_{T} r \exp \left(-\pi \lambda_{T} r^{2}\right)$ and $f_{\rho}(\rho)=2 \pi \lambda_{R} \rho \exp \left(-\pi \lambda_{R} \rho^{2}\right)$, respectively. Thus, averaging over $r$ in (6) yields the association probability with TBS as:

$$
\begin{aligned}
& \mathcal{P}_{A_{T}}=\int_{0}^{\infty} \exp \left(-\pi \lambda_{R}\left(K r^{2}\right)^{\frac{2}{\alpha}} \exp \left(\frac{2 k_{a}(f) r}{\alpha}\right)\right) f_{r}(r) d r \\
& \stackrel{(a)}{=} \int_{0}^{\infty} 2 \pi \lambda_{T} \alpha h^{2 \alpha-1} e^{-\pi \lambda_{T} h^{2 \alpha}} \exp \left(-\pi \lambda_{R} K^{\frac{2}{\alpha}} h^{4} e^{\frac{2 k_{a}(f) h^{\alpha}}{\alpha}}\right) d h, \\
& \stackrel{(b)}{=} \int_{0}^{\infty} 2 \pi \lambda_{T} \alpha h^{2 \alpha-1} e^{-\pi \lambda_{T} h^{2 \alpha}} \sum_{j=0}^{\infty} \frac{\left(-\pi \lambda_{R} K^{\frac{2}{\alpha}} h^{4} e^{\frac{2 k_{a}(f)}{\alpha} h^{\alpha}}\right)^{j}}{j !} d h, \\
& \stackrel{(c)}{=} \sum_{j=0}^{\infty} \frac{\left(-\pi \lambda_{R} K^{\frac{2}{\alpha}}\right)^{j}}{j !} \int_{0}^{\infty} 2 \pi \lambda_{T} z^{\frac{4 j+\alpha}{\alpha}} e^{-\pi \lambda_{T} z^{2}+\frac{2 j k_{a}(f)}{\alpha} z} d z, \\
& =\sum_{j=0}^{\infty} \frac{\left(-\pi \lambda_{R} K^{\frac{2}{\alpha}}\right)^{j}}{j !} \int_{0}^{\infty} 2 \pi \lambda_{T} z^{v_{j}-1} e^{-\beta z^{2}-\eta_{j} z} d z,
\end{aligned}
$$

where (a) is derived by changing variables $r=h^{\alpha}$, (b) follows from expanding the exponential function as $\exp (-x)=$ $\sum_{i=0}^{\infty}(-1)^{i} \frac{x^{i}}{i !}$ ( [13], Eq. 1.211), (c) follows from the variable change $z=h^{\alpha}$, and, finally, Lemma 2 is derived by using the integral identity $\int_{0}^{\infty} x^{\nu-1} e^{-\beta x^{2}-\eta x} d x=$ $(2 \beta)^{-\frac{\nu}{2}} \Gamma[\nu] \exp \left(\frac{\eta^{2}}{8 \beta}\right) D_{-\nu}\left(\frac{\eta}{\sqrt{2 \beta}}\right)$ ( [13], Eq. 3.462).

\section{Appendix C: Proof of Lemma 3}

The distribution of the distance from the tagged BS in the tier $k$ where $k=\{\mathrm{THz}, \mathrm{RF}\}$ can be derived as follows:

$$
\begin{aligned}
f_{\hat{X}_{k}}(\hat{x}) & =\frac{d \operatorname{Pr}\left[\hat{X}_{k}>\hat{x}\right]}{d \hat{x}}=\frac{d \operatorname{Pr}\left[X_{k}>\hat{x} \mid k=n\right]}{d \hat{x}} \\
& =\frac{d \operatorname{Pr}\left[X_{k}>\hat{x}, k=n\right]}{\operatorname{Pr}[k=n] d \hat{x}},
\end{aligned}
$$

where $n \in\{\mathrm{THz}, \mathrm{RF}\}$ is the index of the layer to which a user will associate. $\operatorname{Pr}[k=n]$ is the association probability of a user to tier $k$ as given in Lemma 2. When the user associates to the TBS, the numerator in C.1 can be given as:

$$
\begin{aligned}
& \operatorname{Pr}\left[X_{T}>\hat{x} \mid k=\mathrm{THz}\right]=\operatorname{Pr}\left[X_{T}>\hat{x}, B_{T} P_{r}^{\mathrm{THz}}>P_{r}^{\mathrm{RF}}\right], \\
& =\int_{\hat{x}}^{\infty} \operatorname{Pr}\left[B_{T} P_{r}^{\mathrm{THz}}>P_{r}^{\mathrm{RF}}\right] f_{X_{T}}(x) d x, \\
& \stackrel{(a)}{=} \int_{\hat{x}}^{\infty} 2 \pi \lambda_{T} x e^{-\pi \lambda_{R} K^{2 / \alpha} x^{4 / \alpha} e^{2 k_{a}(f) x / \alpha}-\pi \lambda_{T} x^{2}} d x,
\end{aligned}
$$

where (a) is derived by substituting $\operatorname{Pr}\left[P_{r}^{\mathrm{THz}}>P_{r}^{\mathrm{RF}}\right]$ provided in Appendix $\mathbf{B}$, and $f_{X_{T}}(x)=2 \pi \lambda_{T} x \exp \left(-\pi \lambda_{T} x^{2}\right)$.

\footnotetext{
${ }^{4}$ User association to a BS is a slowly varying process that relies on longterm channel propagation factors such as path-loss and shadowing.
}

Now substituting (C.2) in C.1 results in $f_{\hat{X}_{T}}(\hat{x})$. Likewise, when the user associates to the RF layer, we have:

$$
\begin{aligned}
& \operatorname{Pr}\left[X_{R}>\hat{x} \mid k=\mathrm{RF}\right]=\operatorname{Pr}\left[X_{R}>\hat{x}, P_{r}^{\mathrm{RF}}>B_{T} P_{r}^{\mathrm{THz}}\right], \\
& =\int_{\hat{x}}^{\infty} \operatorname{Pr}\left[P_{r}^{\mathrm{RF}}>B_{T} P_{r}^{\mathrm{THz}}\right] f_{X_{R}}(x) d x, \\
& =\int_{\hat{x}}^{\infty} \operatorname{Pr}\left[\pi r^{2} \exp \left(k_{a}(f) r\right)>K x^{\alpha}\right] f_{X_{R}}(x) d x, \\
& \stackrel{(a)}{\approx} \int_{\hat{x}}^{\infty} \operatorname{Pr}\left[r>\left(\frac{K x^{\alpha}}{\pi}\right)^{\frac{1}{2+\mu}}\right] f_{X_{R}}(x) d x, \\
& =\int_{\hat{x}}^{\infty} 2 \pi \lambda_{R} x \exp \left(-\pi \lambda_{T}\left(\frac{K x^{\alpha}}{\pi}\right)^{\frac{1}{2+\mu}}-\pi \lambda_{R} x^{2}\right) d x,
\end{aligned}
$$

where (a) is derived by approximating $r^{2} \exp \left(k_{a}(f) r\right)$ with $r^{2+\mu}$, and $\mu$ is a correcting factor. That is, when $k_{a}(f)>0.1$ than $\mu=$ $2+\frac{10 k_{a}(f)}{1+2 k_{a}(f)}$, otherwise $\mu=2+\frac{15 k_{a}(f)}{1+10 k_{a}(f)}$. Finally, substituting C.3 in C.1 results $f_{\hat{X}_{R}}(\hat{x})$.

\section{REFERENCES}

[1] A.-A. A. Boulogeorgos, E. N. Papasotiriou, and A. Alexiou, "Analytical performance assessment of THz wireless systems," IEEE Access, vol. 7 , pp. 11436-11453, 2019.

[2] Y. Nakasha, S. Shiba, Y. Kawano, and T. Takahashi, "Compact terahertz receiver for short-range wireless communications of tens of Gbps," FUJITSU Sci. Tech. Journal, vol. 53, no. 2, pp. 9-14, 2017.

[3] J. Kokkoniemi, J. Lehtomäki, and M. Juntti, "Stochastic geometry analysis for mean interference power and outage probability in $\mathrm{THz}$ networks," IEEE Trans. on Wireless Commun., vol. 16, no. 5, pp. 3017 3028, 2017.

[4] V. Petrov, M. Komarov, D. Moltchanov, J. M. Jornet, and Y. Koucheryavy, "Interference and sinr in millimeter wave and terahertz communication systems with blocking and directional antennas," IEEE Trans. on Wireless Commun., vol. 16, no. 3, pp. 1791-1808, 2017.

[5] C. Chaccour, R. Amer, B. Zhou, and W. Saad, "On the reliability of wireless virtual reality at terahertz $\mathrm{THz}$ frequencies," in 10th IFIP Intl. Conf. on New Technologies, Mobility and Security (NTMS), 2019.

[6] K. Ntontin and C. Verikoukis, "Toward the performance enhancement of microwave cellular networks through THz links," IEEE Trans. on Vehicular Technology, vol. 66, no. 7, pp. 5635-5646, 2016.

[7] D. Liu, L. Wang, Y. Chen, M. Elkashlan, K.-K. Wong, R. Schober, and L. Hanzo, "User association in 5G networks: A survey and an outlook," IEEE Commun. Surveys \& Tut., vol. 18, no. 2, pp. 1018-1044, 2016.

[8] S. Mumtaz, J. M. Jornet, J. Aulin, W. H. Gerstacker, X. Dong, and B. Ai, "Terahertz communication for vehicular networks," IEEE Trans. on Vehicular Technology, vol. 66, no. 7, pp. 5617-5625, 2017.

[9] C. Chaccour, M. N. Soorki, W. Saad, M. Bennis, and P. Popovski, "Can terahertz provide high-rate reliable low latency communications for wireless VR?" arXiv preprint arXiv:2005.00536, 2020.

[10] M. Di Renzo, "Stochastic geometry modeling and analysis of multi-tier millimeter wave cellular networks," IEEE Trans. on Wireless Commun., vol. 14, no. 9, pp. 5038-5057, 2015.

[11] J. Gil-Pelaez, "Note on the inversion theorem," Biometrika, vol. 38, no. 3-4, pp. 481-482, 1951.

[12] H. Tabassum and E. Hossain, "Coverage and rate analysis for co-existing rf/vlc downlink cellular networks," IEEE Transactions on Wireless Communications, vol. 17, no. 4, pp. 2588-2601, 2018.

[13] I. S. Gradshteyn and I. M. Ryzhik, Table of integrals, series, and products. Academic press, 2014.

[14] J. M. Jornet and I. F. Akyildiz, "Channel modeling and capacity analysis for electromagnetic wireless nanonetworks in the terahertz band," IEEE Trans. on Wireless Commun., vol. 10, no. 10, pp. 3211-3221, 2011.

[15] L. S. Rothman, I. E. Gordon, A. Barbe, D. C. Benner, P. F. Bernath, M. Birk, V. Boudon, L. R. Brown, A. Campargue, J.-P. Champion et al., "The HITRAN 2008 molecular spectroscopic database," Journal of Quantitative Spectroscopy and Radiative Transfer, vol. 110, no. 9-10, pp. 533-572, 2009.

[16] T. Bai, R. Vaze, and R. W. Heath, "Analysis of blockage effects on urban cellular networks," IEEE Trans. on Wireless Commun., vol. 13, no. 9, pp. 5070-5083, 2014. 